\title{
PERCEPÇÕES DOS ATORES SOCIAIS SOBRE O ATENDIMENTO PRESTADO PELO LABORATÓRIO ÓPTICO DO PROGRAMA DE ASSISTÊNCIA PRIMÁRIA DE SAÚDE ESCOLAR (PROASE) NO MUNICÍPIO DE RIBEIRÃO PRETO
}

\author{
Maria das Graças Carvalho Ferriani ${ }^{1}$ \\ Giovanna Teresinha Candido ${ }^{2}$
}

Ferriani MGC, Candido GT. Percepções dos atores sociais sobre o atendimento prestado pelo laboratório óptico do programa de assistência primária de saúde escolar (PROASE) no município de Ribeirão Preto. Rev Latino-am Enfermagem 2001 novembro-dezembro; 9(6):42-8.

O presente estudo objetivou avaliar o atendimento prestado pelo Laboratório Óptico do PROASE, segundo a percepção dos familiares e professores dos escolares que adquiriram óculos no período de 1991 a 1995, no município de Ribeirão Preto. Utilizamos para a análise dos dados a proposta indicada para análise de conteúdo e para atingirmos os significados manifestos no material qualitativo, utilizamos a técnica de análise temática. Concluímos que os atores sociais manifestam de forma positiva a importância do programa e deixam evidente que 0 PROASE não só na área de oftalmologia vem trazendo contribuições relevantes para saúde das crianças em idade escolar.

DESCRITORES: enfermagem, estudantes, oftalmologia

\section{PERCEPTIONS OF SOCIAL ACTORS CONCERNING THE CARE DELIVERED BY THE OPTICAL LABORATORY IN THE PROGRAM OF PRIMARY CARE TO SCHOOLCHILDREN (PROASE) IN THE CITY OF RIBEIRÃO PRETO}

This study aimed at evaluating the care delivered by the Optical Laboratory of the Program of Primary Care to Schoolchildren (PROASE) according to the perceptions of relatives and teachers of children who had acquired glasses from 1991 to 1995 in the municipality of Ribeirão Preto. The content analysis technique was used for data analysis and, in order to observe the meanings shown in the qualitative material, the thematic analysis technique was utilized. It was concluded that that the social actors showed a positive attitude concerning the importance of the program and clearly expressed that PROASE has given relevant contribution to the health of schoolchildren.

KEY WORDS: nursing, schoolchildren, ophthalmology

\section{PERCEPCIONES DE LOS ACTORES SOCIALES SOBRE LA ATENCIÓN DADA POR EL LABORATORIO ÓPTICO DEL PROGRAMA DE ATENCIÓN PRIMARIA DE SALUD ESCOLAR (PROASE) EN EL MUNICIPIO DE RIBEIRÃO PRETO}

El presente estudio tuvo como objetivo evaluar la atención prestada por el Laboratorio Óptico del PROASE, según la percepción de Ios familiares y profesores de los escolares que adquirieron gafas, en el período de 1991 a 1995, en el municipio de Ribeirão Preto. Utilizamos para el análisis de los datos la propuesta indicada para el análisis de contenido y para alcanzar los significados dados en el material cualitativo, utilizamos la técnica de análisis temático. Concluimos que los actores sociales manifiestan de forma positiva la importancia del programa y hacen evidente que el PROASE ha traído contribuciones relevantes para la salud de los niños en edad escolar y no solo en el área de oftalmología

DESCRIPTORES: enfermería, estudiantes, oftalmología

\footnotetext{
${ }^{1}$ Enfermeiro, Professor Titular da Escola de Enfermagem de Ribeirão Preto da Universidade de São Paulo, Centro Colaborador da OMS para o desenvolvimento da pesquisa em enfermagem, e-mail: caroline@eerp.usp.br; ${ }^{2}$ Aluna de Graduação da Escola de Enfermagem de Ribeirão Preto da Universidade de São Paulo, Centro Colaborador da OMS para o desenvolvimento da pesquisa em enfermagem, Bolsista PIBIC/CNPq
} 
INTRODUÇÃO

Calcula-se que 11,4 milhões de pessoas nos Estados Unidos têm algum tipo de perturbação visual. Dentre esses, aproximadamente 1,4 milhão tem a visão reduzida, o que acaba interferindo em sua vida normal. Indica-se que o tratamento da baixa visão deve ser iniciado em qualquer estágio em que o paciente sinta dificuldade em realizar as tarefas visuais costumeiras ${ }^{(1)}$.

O olho é o órgão responsável pela visão. E esta se torna possível pela conversão dos raios luminosos em impulsos nervosos. Quando o olho apresenta erros de refração faz-se necessário o uso de óculos ou lentes para a correção desses defeitos. Os erros de refração incluem a miopia (visão de perto), a hiperopia (visão de longe), a anisometria (focalização desigual nos dois olhos), astigmatismo (focalização assimétrica) e presbiopia (incapacidade de mudar de foco).

Os erros de refração e seu tratamento são mais conhecidos quando relacionados ao processo de acomodação. A acomodação é produzida quando os músculos ciliares se contraem, aumentando a curvatura do cristalino. Isto faz com que o poder refratário do olho aumente (acomodação) e resulte em deixar o olho capaz de focalizar sobre os objetos próximos. Quando os músculos ciliares relaxam, 0 poder refratário do olho encontra-se em seu mais baixo grau possível.

Quando o olho normal é focalizado para longe, sem acomodação, ocorre a emetropia. 0 olho enxerga claramente objetos à distância sem esforço e, usando a acomodação, pode focalizar objetos próximos.

Nas condições normais, o desenvolvimento da visão em toda sua integridade só se completa por volta dos 5 a 6 anos de idade. Sendo assim qualquer distúrbio da visão na primeira infância terá conseqüências no desenvolvimento psicossocial da criança, daí a importância em realizar-se exames preventivos desde cedo, na creche ou na pré-escola.

A maioria das deficiências visuais que surgem antes da idade escolar passa despercebida na família. A criança pode não se queixar pôr não saber o quanto deve enxergar, ou abster-se de atividades que exijam maior esforço visual. A falta de conhecimento dos pais dificulta a identificação do problema.

Muitos estudos, ressaltam a importância da avaliação e 0 tratamento da criança, como parte da aprendizagem escolar e a saúde visual do adulto ${ }^{(2-4)}$. A conclusão é que a solução ou minimização de distúrbios oftalmológicos em escolares dependem, significativamente, do conjunto de pessoal de ensino, da família, da comunidade e pessoal da saúde ${ }^{(5)}$.

\section{A PRÁTICA DO PROGRAMA DE SAÚDE ESCOLAR NO MUNICÍPIO DE RIBEIRÃO PRETO EM RELAÇÃO À SAÚDE OCULAR}

O Programa de Assistência Primária de Saúde Escolar (PROASE) atua na rede pública de ensino de Ribeirão Preto, desde 1985, através de um convênio firmado entre a Escola de Enfermagem de Ribeirão Preto-USP e a Prefeitura Municipal de Ribeirão Preto, tendo como interveniente a Secretaria Municipal da Saúde.

O objetivo deste Programa é promover a atenção integral à saúde da criança e do adolescente em idade escolar, compreendendo ações de promoção, preservação e recuperação da saúde, através da rede de serviços e da ação integrada entre os setores Saúde e Educação, além de outros programas e instituições, contribuindo assim para a melhoria da saúde e conseqüentemente do processo de desenvolvimento e formação integral da criança e do adolescente.

Dentre os diversos subprojetos existentes, desenvolve-se um Programa de Oftalmologia Sanitária Escolar, que atua na prevenção de danos causados pelos problemas escolares, a fim de garantir condições de bem estar físico, psíquico e social à criança.

Desde a sua implantação no município, o PROASE tem se preocupado em desenvolver, junto às escolas, um programa de Oftalmologia Sanitária Escolar, que se estabelece através da atuação do enfermeiro com o apoio do médico oftalmologista vinculado ao programa.

O PROASE promove cursos aos auxiliares de enfermagem que atuam nas escolas e aos professores, visando capacitá-los para realizarem os testes de triagem oftalmológica dos escolares. Todas as crianças de $1^{\text {a }}$ série ingressantes na escola são submetidas ao teste de acuidade visual e se necessário, encaminhados aos oftalmologistas do PROASE. Identificados possíveis problemas visuais atua-se intervindo junto às famílias no sentido de orientá-las a utilizar os recursos disponíveis da comunidade.

Através de estudos e de nossa vivência no programa, constatamos que estes procedimentos estavam ocorrendo, entretanto a família, muitas vezes, parava na $1^{\text {a }}$ consulta, deixando de adquirir os óculos pôr questões econômicas, ou aguardando a "Campanha dos Óculos Escolar", que era promovida pelo governo do Estado de São Paulo.

Diante das questões levantadas, os coordenadores do PROASE - docentes da Escola de Enfermagem de Ribeirão PretoUSP - elaboraram um projeto para implantação de um Laboratório Óptico. Este projeto foi enviado à Fundação de Assistência ao Estudante (FAE), tendo sido aprovado em 1990, e resultou em um convênio entre este órgão e a Escola de Enfermagem de Ribeirão Preto-USP. 
Os recursos materiais para confecção/montagem gratuita de óculos para escolares vinha sendo repassado pela FAE até janeiro de 1996, quando houve a extinção deste órgão. O Laboratório Óptico do PROASE foi inaugurado em 1991 e vem fornecendo óculos aos escolares que não têm condições de adquiri-los em serviços particulares. Cabe ressaltar que 0 valor de um óculos (lente e armação) nestes serviços particulares corresponde, em média, ao valor do salário mínimo vigente no país. O referido Laboratório conta com (02) dois auxiliares de enfermagem funcionários da Secretaria Municipal da Saúde, treinados para desenvolver atividades técnicas de teste de acuidade visual e montagem dos óculos.

Diante do exposto, emerge a preocupação de como vem ocorrendo 0 atendimento prestado à população de Ribeirão Preto pelo Laboratório Óptico do PROASE. Portanto, o objetivo deste estudo é conhecer e analisar a percepção dos familiares e professores dos escolares que adquiriram óculos no período de 1991 a 1995 no município de Ribeirão Preto, em relação ao atendimento prestado pelo referido Laboratório.

\section{MÉTODOS}

No presente estudo utilizamos a pesquisa qualitativa ${ }^{(6)}$ como aquela capaz de "aprofundar a complexidade de fenômenos, fatos e processos particulares e específicos de grupos, mais ou menos delimitados em extensão, capazes de serem abrangidos intensamente". Entretanto, durante o processo de estudo utilizamos também, dados quantitativos para melhor dimensionar a validade do objeto de estudo.

Primeiramente realizamos o levantamento dos Relatórios Bimestrais do Laboratório Óptico do PROASE, encaminhados à FAE, com o intuito de conhecermos a quantidade de óculos confeccionados e distribuídos desde a sua implantação, ou seja, de 1991 a 1995, no município de Ribeirão Preto. Concomitante ao levantamento foi realizado observação das atividades realizadas no cotidiano profissional dos auxiliares de enfermagem que atuam no Laboratório Óptico.

Foram entrevistadas 12 famílias dos escolares que adquiriram óculos e 12 professores das escolas que as crianças pertenciam. Na escolha da amostra utilizamos as recomendações ${ }^{(7)}$, com relação aos critérios básicos para amostragem em pesquisa qualitativa, ou seja, o critério não é numérico, preocupando-se com o aprofundamento e abrangência da compreensão, seja de "um grupo social, de uma organização, de uma instituição, de uma política ou de uma representação". Nessa perspectiva, estabelecemos o limite pela repetição das consultas e reincidência das informações.

Realizamos visitas domiciliares para entrevista com as famílias e nas escolas com os professores. As mesmas ocorreram durante os meses de março e abril de 1997, em dias úteis de trabalho (de segunda a sexta-feira) no período da manhã. As visitas nas escolas foram realizadas nos meses de agosto e setembro de 1997, em dias úteis de trabalho, no período da manhã e/ou à tarde, durante o horário letivo de aulas (segunda a sexta-feira), de acordo com a disponibilidade dos professores.

Durante as visitas aplicamos um instrumento de coleta de dados (A 1 e 2) realizando entrevistas do tipo semi-estruturada.

Somente consideramos como visita realizada aquela em que havia um dos familiares presente na residência, ou seja, não foram entrevistadas as famílias nas quais não se encontrava 0 responsável pela criança. Todas as entrevistas foram gravadas, conforme Esclarecimento Livre Esclarecido dos familiares e dos professores e da Coordenadora do PROASE. Posteriormente, realizamos a transcrição dos dados, tomando-se o cuidado em manter a originalidade dos relatos a serem analisados. As entrevistas tiveram duração média de 30 minutos e foram realizadas por uma das pesquisadoras envolvida no projeto.

\section{ANÁLISE DOS DADOS}

$\mathrm{Na}$ busca de atingirmos os significados manifestos no material coletado, utilizamos a técnica de análise temática. A noção de tema está ligada a uma afirmação a respeito de determinado assunto. Ela comporta feixe de relações e pode ser graficamente apresentada através de uma palavra, uma frase, um resumo.

Em relação a análise de dados utilizamos a proposta de Bardin ${ }^{(8)}$ para análise de conteúdo. Entendemos que esta análise se aplica a qualquer comunicação (oral, visual, gestual) reduzida a um texto ou documento.

Resultados do trabalho

De acordo com a Figura 1, observamos que durante 0 período de 1993 a 1995, foram distribuídos um total de 1385 (100\%) óculos para o município de Ribeirão Preto e um total de 5051 (100\%) óculos para os municípios conveniados, sendo que a distribuição anual para Ribeirão Preto manteve-se em média 33\% do total de óculos, porém houve um aumento significativo do número de óculos distribuídos para os municípios conveniados que inicialmente era de 12,9\% em 1993, 21,8\% em 1994, passando para 65,1\% em 1995. Estes dados apontam que houve um aumento significativo na procura de óculos gratuito para escolares no Laboratório Óptico por parte dos outros municípios. Isto indica que os municípios conveniados passaram a utilizar mais os serviços prestados pelo referido Laboratório. 
No que se refere a cota de óculos fornecida pela FAE neste período, podemos verificar pela Figura 2, que houve um aumento de 6,82\% para 46,59\% para Ribeirão Preto, como para os municípios conveniados que foi de $2,78 \%$ para $87,18 \%$ óculos/ano, ou seja, a procura pelo serviço do Laboratório Óptico aumentou cerca de $81 \%$, bem como convênio de outros municípios cerca de $58 \%$.

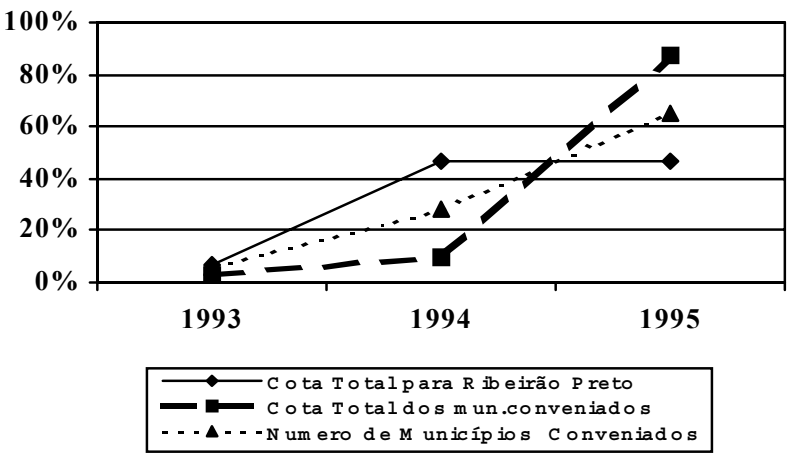

Figura 1 - Diagrama do total de Óculos Distribuídos pelo Laboratório Óptico para Ribeirão Preto e Outros municípios conveniados nos anos de 1993, 1994 e 1995

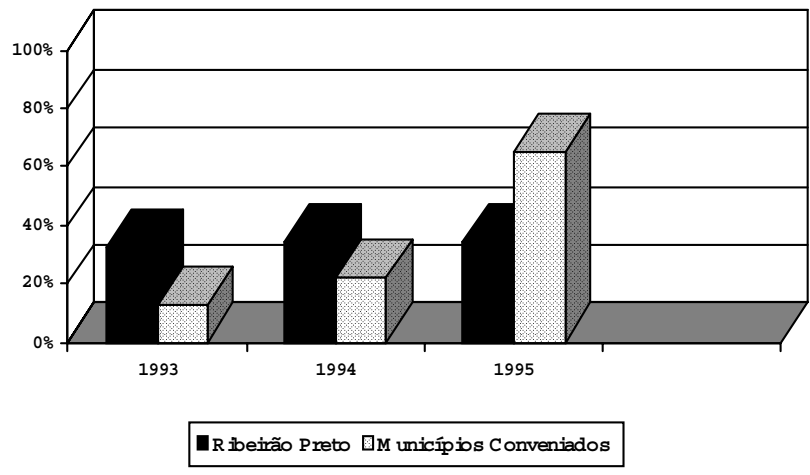

Figura 2 - Distribuição da Cota de óculos para o município de Ribeirão Preto, Cota para todos os municípios e Número de Municípios Conveniados nos anos de 1993, 1994 e 1995

O aumento do número de municípios conveniados com 0 Laboratório Óptico do PROASE deveu-se ao fechamento de outros Centros de Referência, no Estado de São Paulo, com os quais a FAE mantinha convênio. Portanto, Ribeirão Preto ficou sendo o único local de referência para esses municípios, para confecção e distribuição de óculos de grau.

\section{Caracterização dos atores sociais}

Em relação aos familiares que participaram da pesquisa, do total de 12 entrevistas realizadas, 10 foram com mães dos escolares e outras duas com avós. As famílias investigadas apresentam renda familiar média de 4 (quatro) salários mínimos.

No que concerne ao nível de escolaridade, as entrevistadas possuem, em média, $01^{\circ} \mathrm{grau}$ incompleto, sendo que uma das avós é analfabeta. Com exceção de uma avó, que é comerciante, todas as outras apresentam ocupação do Lar.
Os escolares apresentam idade entre 07 e 15 anos, maioria do sexo feminino, sendo somente 02 masculinos, e a escolaridade variando entre $1^{\circ}$ série do primeiro grau a $1^{\circ}$ ano do colegial.

Quanto aos professores, estes apresentam idade entre 32 a 52 anos, todos do sexo feminino, com tempo de profissão entre $6 \mathrm{e}$ 19 anos.

No sentido de melhor compreendermos os dados de nosso estudo, escolhemos os seguintes núcleos temáticos de análise que contemplavam as falas dos sujeitos sociais entrevistados: sinais e sintomas; atendimento oftalmológico e aquisição de óculos.

a) Sinais e Sintomas

Podemos evidenciar nas falas dos atores sociais que os sinais e sintomas que uma criança apresenta em relação a dificuldade visual não são desconhecidos nem por parte da famílias como dos professores.

Através dessas falas, constamos que a prática do Programa de Saúde Escolar desenvolvido no município de Ribeirão Preto, muito tem contribuído de forma positiva junto as famílias e as escolas em relação a detecção de problemas oftalmológicos.

Como exemplo citamos os seguintes fragmentos de suas falas: Percebo que esta parte da oftalmologia nas crianças que tem dificuldade é muito importante, as vezes a criança não acompanha...(EP5). Ela reclamava de dificuldades para ver na lousa e a professora falou que era óculos que ela precisava...(EF3).

De acordo com a proposta do PROASE, este vem oferecendo cursos para professores sobre os principais sinais e sintomas de distúrbios oftalmológicos, visando capacitá-los, objetivando detectar os problemas visuais precocemente. Concordamos com vários autores como ${ }^{(2-5)}$ que aludem para a importância da avaliação e o tratamento da criança como parte da aprendizagem do adulto e a própria saúde visual.

Depreendemos ainda, nas falas que essas estratégias têm favorecido as famílias e professores um melhor entendimento de como lidar com as questões relacionadas a Saúde Escolar.

b) Aquisição de Óculos

Evidenciamos nos depoimentos dos sujeitos sociais, o valor atribuído ao Programa de Saúde Escolar por ter possibilidade de fornecer a população o óculos que consideram caro e deixam explicito em suas falas: Sei que óculos custa caro, não sei quanto mais sei que é caro, eu não tenho condições de comprar, ainda bem que ela ganhou (EF1). Graças a Deus que ela ganho esse óculos eu não ia te dinheiro para compra (EF1). Ajudou muito ela te recebido estes óculos, nunca deu problema, e ela ta usando direitinho (EF2). ....Nossa! eu fico muito agradecida dela te ganhado 
óculos, do jeito que eu ganho pouco, ruum! Não dava nunca pra compra (EF3). Bom, achei bom assim não preciso gasta (EF4). Custa caro sim, hoje deve tá assim uns cento e vinte reais (EF7). Eu acho importante, não é de graça, porque eu pago imposto e pelo menos isso tem que dar esse governo, mas tem uns que nem isso não dá ...(EF9).

Percebemos também que a população conhece seus direitos, entendendo não ser de graça a aquisição de óculos pelo Laboratório Óptico, tendo em vista que pagam pelo seus direitos.

Historicamente é sabido que a questão da cidadania é construída e conquistada pela luta e participação do indivíduo na sociedade, que assim conquista direitos e diminui as diferenças sociais. "Cidadania é qualidade social de uma sociedade organizada sob forma de direitos e deveres majoritariamente reconhecidos" ${ }^{\prime \prime 9)}$.

Outra questão que evidenciamos nas falas é que o atendimento prestado pelo referido laboratório às vezes torna-se parcial. Este fato ocorre porque todo o material (armação e lentes) fornecido pela Fundação de Apoio ao Estudante (FAE) era padronizado. Os critérios para padronização destes materiais foram estabelecidos pela FAE a partir da experiência adquirida com outros programas similares, levando em conta ainda, aspectos burocráticos e técnicos. Isto implica em acúmulo daquelas lentes menos solicitadas e por outro lado falta daquelas mais solicitadas. Segue anexa planilha com as lentes mais solicitas.

O Laboratório Óptico trabalha apenas com lentes de cristal incolor, esféricas até 6.00 dioptrias e lentes cilíndricas até 3.00 dioptrias, não possuindo, portanto, lentes com graus acima dos descritos. Portanto o Laboratório não trabalha: com lentes 0.25 dioptrias ou frações de 0.25 dioptrias; com lentes bifocais; lentes acrílicas ou endurecidas; lentes especiais; lentes coloridas.

Quando a lentes solicitadas se encontra em falta, os funcionários do referido laboratório fornecem somente a armação do óculos e indicam um Serviço de Óptica particular para que as lentes sejam adquiridas. Esta indicação é realizada mediante pesquisa prévia para cotação do preço de lentes, sendo indicada o que oferecer o valor mais acessível para familiares dos escolares. Quando precisei de novo fui, mas não tinha a lente...(EF5). Sei que é caro porque do outro menino eu precisei compra as lentes só peguei a armação lá no laboratório, porque não tinha a lente lá, então eu comprei (EF5).

Cabe salientar que uma das cinco diretrizes básicas da FAE é a utilização de tecnologia simplificada. Os equipamentos, materiais e instrumental adquiridos para o programa devem corresponder ao nível de complexidade dos serviços, privilegiando a adoção de tecnologia simples. Por outro lado, o programa tem procurado atender esta diretriz da FAE referente a material simplificado, mas sempre avaliando a eficiência dos mesmos. Acrescenta ainda, que a preocupação maior do PROASE tem sido aprimorar a assistência ao escolar e com isso vem questionando as ações e o instrumental, propondo novas idéias e técnicas, para um melhor resultado dessa assistência ${ }^{(10)}$.

c) Atendimento Oftalmológico

Percebemos que 0 atendimento à população se faz de forma indiscriminada, atendendo a todas as solicitações que se apresentam. 0 único critério estabelecido é que a criança seja préescolar ou escolar, obedecendo à finalidade do Laboratório Óptico do Programa de Saúde Escolar, ou seja, atendimento ao escolar. A divulgação e as indicações para procura do Laboratório são feitas por profissionais da saúde e educação (médicos, enfermeiras, professores) como também por parte da própria população. Foi uma colega minha que me falou que dava óculos lá nesse laboratório, ai eu fui lá (EF1)....Foi a doutora do posto, quando fez a receita já mandou eu i lá com a cartinha vê se tinha óculos (EF2). Olha a minha vizinha que falou que uma criança da classe da filha dela tinha ganhado óculos, ai eu pedi pra ela perguntar, liguei lá e fui depois (EF5). Quando eu vi que ele precisava de óculos eu já fui direto no laboratório, porque a professora já tinha me falado (EF6). Quem falou pra mãe dela do Laboratório foi a professora da escola falou que era pra ver se conseguia, que era de graça , ai eu fui lá (EF7). Foi minha vizinha a filha dela trabalha no posto e ela me deu o endereço do laboratório (EF8). Fizeram teste na escola, ela trouxe uma cartinha, foi na consulta e depois foi lá pegar o óculos (EF11).

A procura pelo serviço do Laboratório Óptico se da tanto por parte das famílias que utilizam o Sistema Único de Saúde (Unidades Básicas) como também por aquelas que têm convênio médico. Muitas vezes a procura por consultas com oftalmologistas da rede básica de saúde ultrapassa o número de consultas disponíveis nos locais de atendimento, dificultando 0 acesso dos escolares ao atendimento oftalmológico. Anteriormente, o PROASE contava com um número de consultas oftalmológicas exclusivas para o atendimento dos escolares, atualmente isto não ocorre, fator que dificultou o acesso dos escolares às consultas, tendo estes que esperar por uma vaga na rede básica que é oferecida à população como um todo. No entanto mesmo que as famílias procurem os convênios para agilizar a consulta acabam procurando o Laboratório Óptico para aquisição de óculos. Eu levei ele no postinho aqui da vila, mas como ia demorar muito pra marcar levei no convênio da firma do meu marido (EF6). Foi o médico do CSU que falou do Laboratório (EF3). Tenho convênio médico...foi lá que levei ela no médico que falou dos óculos pra ela (EF1).

Além da notável quantidade de atendimentos que se evidencia através dos dados numéricos (Figura 1), constatamos através das falas dos atores sociais que estes apresentam também valor qualitativo no que se refere aos atendimentos oferecidos à população e aos benefícios que o Programa de Saúde Ocular do PROASE traz para o aluno. 
Alguns autores ${ }^{(11-13)}$, que trabalham com avaliação de

serviço, consideram que aceitação de serviço inclui vários aspectos, dentre os quais salientamos a expectativa das pessoas referente à qualidade da assistência, e que para o paciente aceitar o serviço, ele deve ter confiança na competência técnica e no tratamento humano dispensado pelo profissional que assiste.

Estes autores inferem que os fatores mais importantes que influenciam na percepção dos pacientes são: "interesse pessoal do médico pelo paciente, acessibilidade, bons médicos, acompanhamento por pessoal treinado, solicito, privacidade e informações dos médicos."

O confronto entre os fatores citados por estes autores e os identificados nas falas dos atores sociais, na observação e na documentação analisada, revela os mesmos elementos valorizados pelos usuários do Laboratório Óptico como podemos observar: Eu fui bem tratada. Bem, foi tudo bem. Nunca teve nada (EF1).Acho de extrema importância este Programa não só em relação a oftalmologia como também, as outras atividades que são desenvolvidas (EP1). Quando fui lá, fui bem atendida, não demorou, fui bem tratada (EF2). Percebo que de alguns anos pra cá, tem se dado mais importância à saúde das crianças (EP2). As escolas ganharam muito com a implantação deste programa...aqui o aluno é bem assistido...(EP4). É diferenciada e o programa é que faz a diferença (EP4). Fui, me trataram bem sim. Acho muito bom esse serviço... (EF7). Bom... Bom...(EF8). Hoje com este programa a atenção se volta mais para as questões da saúde. E para nós professores, isto é muito gratificante, porque o aluno saudável rende mais (EP2). Bem me trataram bem, não tenho o que reclamar (EF3). Fui bem atendida, as duas vezes que eu fui lá tava aquela moça, ela ma trata bem sim (EF5).

Apreendemos ainda nas falas questões relevantes que retratam a importância da relação usuário e trabalhadores de saúde. 0 autor ${ }^{(14)}$ atenta para o modo como os trabalhadores de saúde se relacionam com o seu principal objeto de trabalho - a vida e o sofrimento dos indivíduos e da coletividade - não basta corrigirmos procedimentos organizacionais e financeiros das instituições de saúde. Entende que um dos grandes desafios é a busca de um outro modo de operar o trabalho em saúde e de construir a relação do trabalhador com os usuários, efetivos, potenciais, do sistema de atenção à saúde sem se esquecer a importância que há nisto. A busca de uma relação mais solidária entre os próprios trabalhadores do ponto de vista de seu desempenho técnico e da construção de um trabalho coletivo.

Acrescenta ainda, que temos que colocar todas as opções tecnológicas de que dispomos em termos de conhecimento, de saber, a serviço do usuário. Temos que primar pela cidadania da assistência à saúde, reconstruindo a nossa dignidade e a do paciente, no campo das ações de saúde.

\section{CONSIDERAÇÕES FINAIS}

Em nosso estudo encontramos, como fatos relevantes 0 número de óculos distribuídos durante o período de 1993 a 1995 que correspondeu a um total de 1.385 para o município de Ribeirão Preto e um total de 5.051 para outros municípios conveniados. Outro aspecto importante, é que houve um aumento da cota de Ribeirão Preto de 550 óculos/anos para 3.762, bem como para os outros municípios de 6.915 para 216.777 óculos/ano.

Em relação ao núcleo temático "sinais e sintomas" a percepção das famílias e professores em relação aos sinais e sintomas que uma criança apresenta com dificuldades visuais não são desconhecidos e isto se deve ao fato do Programa preocupar-se em realizar cursos para professores, discutir com os pais sobre os problemas apresentados pelas crianças.

Outro núcleo temático de nossa investigação é "aquisição de óculos". A família valoriza muito o programa por ter esta possibilidade de poder adquirir o óculos que consideram caro e deixam explícito em suas falas esta importância, bem como chamam atenção ser um direito deles, pois pagam seus impostos. Fica evidente também que o Laboratório Óptico não cobre todas as necessidades da população em decorrência de não contar com um número maior das lentes mais utilizadas , pois o órgão fornecedor deste material trabalha com uma padronização de lentes, quanto ao número e da maneira mais simplificada. Acreditamos que com a extinção da FAE, a coordenação do PROASE, através da EERP-USP e Secretaria Municipal de Saúde devem rever essas questões levantadas e propor uma nova forma de aquisição de lentes que a população mais necessite, para atender melhor os escolares.

Quanto ao "Atendimento Oftalmológico" prestado pelo Programa, especificamente no Laboratório Óptico, os atores sociais (professores e familiares) manifestam de forma positiva a importância do programa e deixam evidente que o PROASE não só na área de oftalmologia vem trazendo contribuições relevantes para saúde das crianças em idade escolar. Ao avaliar o PROASE no município de Ribeirão Preto muitos aspectos foram considerados positivos, como: avanços na Vigilância Epidemiológica e Sanitária nas escolas, aumento da cobertura no atendimento aos escolares nas UBSs, entre estes aspectos a oftalmologia sanitária que tem uma ação preventiva na escola, iniciando com o teste de SNELLEN, o reteste, e a referência para esse escolar se, por conseguinte necessitar da consulta médica, bem como o encaminhamento para o Laboratório Óptico. 
ANEXO 1

\section{Roteiro de entrevista com as famílias que utilizam o Laboratório Óptico do PROASE CARACTERIZAÇÃO \\ DATA $\mathrm{N}^{0}$}

INÍCIO TÉRMINO

NOME DA CRIANÇA

ESCOLA

SÉRIE IDADE SEXO

RESPONSÁVEL

ESCOLARIDADE

IDADE

PROFISSÃO OCUPAÇÃO

RENDA EM SALÁRIOS MÍNIMOS

QUANTAS PESSOAS MORAM EM SUACASA?

QUANTOS IRMÃOS?

FREQÜENTAM A ESCOLA?

TEM ALGUÉM NA FAMÍLIA QUE UTILIZA ÓCULOS? (ESPECIFICAR)

\section{QUESTIONÁRIO}

1. QUANTO TEMPO SEU FILHO USA ÓCULOS?

2. TEM OUTRO FILHO QUE USA ÓCULOS?

3. QUEM FALOU PARA A SENHORA QUE SEU FILHO PRECISAVA USAR

ÓCULOS?

4. TEM CONVÊNIO MÉDICO?

5. SABE QUANTO CUSTA UM ÓCULOS?

\section{REFERÊNCIAS BIBLIOGRÁFICAS}

1. Smeltzer FC, Bare BG. Tratado e enfermagem médico cirúrgica.

7. ed. Rio de Janeiro: Guanabara, Koogan; 1993. v.2.

2. Barbosa NLM, Kara N. Desenvolvimento da visão. Crescimento do globo ocular. Clín Pediátr nov. 1980; 4 (2/3):7-10.

3. Zamo CGS. Estudo da função visual de crianças matriculadas em uma escola pública estadual de primeiro grau na cidade de Porto Alegre-RS. [tese]. São Paulo (SP): Escola Paulista de Medicina; 1998. 4.Condemarin M. Maturidade escolar: manual de avaliação e desenvolvimento das funções básicas para 0 aprendizado escolar. 2. ed. Porto Alegre: Artes Médicas; 1989.

5. Temporini ER. Prevenção da cegueira: participação da escola. Rev Bras Saúde Esc 1992 janeiro; 2(1):41-3.

6. Minayo MCS. Quantitativo-qualitativo oposição ou complementariedade? Cad Saúde Pública 1993 jul/set; 9(3):239-62. 7. Minayo MCS. $O$ desafio do conhecimento: pesquisa qualitativa em saúde. 2. ed. São Paulo: Hucitec/ABRASCO;1993.
6. COMO FICOU SABENDO DO LABORATÓRIO ÓPTICO?

7. RETORNOU AO OFTALMOLOGISTA? E AO LABORATÓRIO ÓPTICO? 8. O QUE SIGNIFICA PARA A SENHORA(O) SEU FILHO TER RECEBIDO OCULOS?

9. COMO SE DÁ A RELAĈ̃O ENTRE OS FUNCIONÁRIOS DO LABORATÓRIO ÓPTICO COM A FAMÍLIA EM RELAÇÃO AO LABORATÓRIO ÓPTICO DO PROASE?

\section{ANEXO2}

Roteiro de entrevista com profissionais da área de educação (professores). PARTE I - CARACTERIZAÇÃO DO PROFISSIONAL

DATA $\mathrm{N}^{0}$

INÍCIO_TÉRMINO

TEMPO DE PROFISSÃO

IDADE SEXO

LOCAL DE TRABALHO

TRABALHA EM OUTRO LOCAL? SIM ( ) NÃO ( )

PARTE ॥

1. COMO VOCÊ PERCEBE O PROGRAMA DE SAÚDE ESCOLAR (PROASE) QUE VEM SENDO DESENVOLVIDO NO MUNICÍPIO DE RIBEIRÃO PRETO EM RELAÇÃO A OFTALMOLOGIA E AO LABORATÓRIO ÓPTICO?

2. COMO SE DÁ A RELAÇÃO ENTRE OS PROFESSORES E A FAMÍLIA EM RELAÇÃO AO ENCAMINHAMENTO PARA O LABORATÓRIO ÓPTICO DO PROASE?

8. Bardin L. Análise de Conteúdo. Lisboa: Edições 70; 1977.

9. Demo P. Participação e conquista. São Paulo: Cortez; 1988.

10. Ferriani MGC. A Inserção do enfermeiro na saúde escolar. São Paulo: EDUSP; 1991.

11. Casanueva E. Programa para la capacitón de una cochorte de estudios perinatales: Il evalución de la aceptación de un servício de salud. Bol Of Sanit Panam jul. 1983; 95(1):44-50.

12. Donabedian A. The quality of care - how can et be assessed? J Am Med Assoc Sept 1988; 260(12):1743-8.

13. Fiedler JC. A review of he literature on access and utilization of medical care with special emphasis on rural primary care. Soc Sci Med 1981;15:129-42.

13. Ferriani MGC. Percepções dos atores sociais que utilizam 0 Programa de Assistência Primária de Saúde Escolar (PROASE) no município de Ribeirão Preto: análise crítica. [tese] Ribeirão Preto (SP): Escola de Enfermagem de Ribeirão Preto; 1994.

14. Merhy EE. O capitalismo e a saúde pública: a emergência das práticas sanitárias no Estado de São Paulo. Campinas: Papirus; 1985. 